\title{
Belgeo
}

Revue belge de géographie

3 | 2014

Art(s) \& Espace(s) / Art(s) \& Space(s)

\section{Entre mouvement et ancrages : les spatialités d'artistes mobiles}

Routes and roots: artistic spatialities on the move

\section{Fabien Barthélémy et Camille Boichot}

\section{(2) OpenEdition}

\section{Journals}

Édition électronique

URL : http://journals.openedition.org/belgeo/13317

DOI : 10.4000/belgeo.13317

ISSN : 2294-9135

Éditeur :

National Committee of Geography of Belgium, Société Royale Belge de Géographie

Référence électronique

Fabien Barthélémy et Camille Boichot, « Entre mouvement et ancrages : les spatialités d'artistes mobiles », Belgeo [En ligne], 3 | 2014, mis en ligne le 16 juin 2015, consulté le 19 avril 2019. URL : http://journals.openedition.org/belgeo/13317 ; DOI : 10.4000/belgeo.13317

Ce document a été généré automatiquement le 19 avril 2019

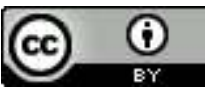

Belgeo est mis à disposition selon les termes de la licence Creative Commons Attribution 4.0 International. 


\title{
Entre mouvement et ancrages : les spatialités d'artistes mobiles
}

\author{
Routes and roots: artistic spatialities on the move
}

\author{
Fabien Barthélémy et Camille Boichot
}

Nous remercions avant tout les artistes qui nous ont consacré du temps et nous ont permis de les accompagner. Nous remercions également nos collègues du programme européen EUBorderscapes pour la richesse des échanges qui ont nourri notre réflexion.

Un grand merci à Florence Troin pour ses précieux conseils cartographiques.

1 L'importance croissante des mobilités dans nos sociétés nous amène à questionner leur rôle dans l'organisation des espaces. Dans cet article, nous interrogeons les relations réciproques entre les formes de mobilité des artistes et des espaces qui peuvent être des lieux, des territoires ou des réseaux. Deux géographes, travaillant tous deux sur les espaces de la création et de la diffusion artistiques, croisent ici leurs observations du monde des arts plastiques et du spectacle vivant pour tenter de comprendre le rôle que les artistes accordent à leur mobilité.

2 L'objectif de cette mise en perspective est de relativiser, à l'aide de données empiriques, l'hypothèse d'une hypermobilité des artistes, valorisée par la métaphore de l'artistenomade (De Morant, 2007). Le nomadisme renvoie à la figure romantique de l'artiste bohème qui survalorise la mobilité en en faisant un idéal de vie (Heinich, 2005 ; Menger, 2002) et minimise l'importance des ancrages qui ponctuent les voyages. Or, s'il est vraisemblable que les artistes constituent une catégorie professionnelle particulièrement mobile, rares sont les études qui analysent ces pratiques et leurs spatialités (AmilhatSzary et al., 2010 ; Deniau, 2011). Pour cela, nous nous positionnons dans la lignée des études de la mobilité qui considèrent cette dernière comme un fait social global intégrant le mouvement en lui-même et plus généralement l'ensemble de ses conditions sociales et culturelles, individuelles et collectives (Kaufmann, 2002 ; Urry, 2000). Notre conception des mobilités s'appuie sur les voyages que les artistes effectuent dans le cadre de leur activité professionnelle mais questionne également la capacité de ce groupe à être 
particulièrement mobile (Kaufmann, 2008). Au-delà des pratiques analysées en termes de formes (fréquence, destinations, portée, etc.) et de logiques (diffusion, création, formation, etc.), il s'agit d'étudier les spatialités induites à la fois par les mobilités et par la dimension artistique. Celles-ci découlent d'une "problématique relationnelle» (Boissière et al., 2010, p. 9) et mettent en jeu les tensions entre l'interne et l'externe, le dedans et le dehors, l'identité et l'altérité, l'ici et l'ailleurs (Bernier, 2013).

Nous choisissons de consacrer cette étude à la mobilité des artistes à l'échelle internationale ${ }^{1}$ que nous observons à partir de données concernant le temps long de la carrière professionnelle comme le temps plus court du projet artistique. Pour ce faire, nous nous appuyons sur le croisement de deux corpus, l'un proposant des données visant à décrire les voyages et l'autre permettant d'en comprendre les ressorts. Le premier corpus est fourni par le FIACRe-international ${ }^{2}$, dispositif de soutien financier à la mobilité internationale des artistes de la Région Rhône-Alpes. Dans un contexte de « dépendance croissante vis-à-vis des ressources publiques » (Cordier, 2007, p. 38), ce dispositif offre un prisme d'observation pertinent pour l'étude des projets artistiques à l'étranger. Nous avons extrait les 46 projets qui ont été retenus lors de la première session de 2014 pour étudier en détail les fiches d'instructions qui recensent les informations relatives aux destinations, à la durée des voyages, aux motifs et objectifs, aux partenaires ainsi que les argumentaires des porteurs de projet. Bien qu'assez restreint quantitativement, cet échantillon est néanmoins représentatif de par la diversité des projets qui le composent en terme d'esthétiques, de destinations, de durée ou de motifs. L'étude de ce corpus a pour but de dégager des profils de voyages et des tendances de pratiques artistiques auxquels font écho les informations analytiques plus approfondies recueillies en entretien et qui constituent le second corpus. Celui-ci est composé d'une cinquantaine d'entretiens semi-directifs réalisés, d'une part, auprès d'artistes plasticiens résidant à Montreuil et à Neukölln ${ }^{3}$ entre 2009 et 2011, et d'autre part, auprès d'artistes du spectacle vivant basés en région Rhône-Alpes en 2013. L'approche biographique menée sur le temps long de la carrière professionnelle permet de comprendre l'importance accordée aux pratiques de mobilité en replaçant celles-ci dans l'ensemble de leur parcours.

Le croisement des observations nous amène à montrer des récurrences dans les pratiques et les spatialités artistiques liées à la mobilité qui vont au-delà des clivages entre disciplines ${ }^{4}$. Nous poserons tout d'abord les termes des débats théoriques et sociétaux dans lesquels la question des mobilités artistiques se situe. Nous examinerons ensuite les spatialités d'artistes mobiles construites autour de différentes articulations entre les espaces de la mobilité et les espaces de l'ancrage, privilégiant tour à tour le voyage en luimême, les lieux de destination et les réseaux. Enfin, nous observerons que ces spatialités artistiques mobiles se manifestent à travers les œuvres en intégrant, par l'esthétique, l'altérité.

\section{Les artistes au cœur de sociétés mobiles}

\section{Les artistes dans le paradigme des mobilités}

5 En un siècle, le monde a connu une accélération considérable des mobilités en parallèle à l'accélération des processus de globalisation. La mobilité occupe aussi par conséquent une place de plus en plus centrale dans les sciences sociales (Cresswell, 2006). L'étude de la mobilité s'est progressivement affirmée comme un champ à part entière, après que $\mathrm{W}$. 
Zelinsky (1971) a proposé le concept de «transition mobilitaire » pour mettre en évidence le lien entre degrés d'évolution des sociétés et régimes de mobilité. Malgré son caractère notablement positiviste, le parallèle entre transition démographique et transition mobilitaire est intéressant: il interroge les conditions de diffusion des pratiques de mobilité au sein d'une société et à l'extérieur de celle-ci. L'approche des mobilités dans un contexte social global est fondatrice et marque fortement les travaux récents.

Considérer les sociétés non plus seulement à partir d'un point de vue sédentaire dominant (Retaillé, 2009), apparaît ainsi comme une nécessité. Cela implique non seulement de prendre en compte les multiples circulations et liens qui caractérisent toute organisation sociale mais aussi les interactions et les systèmes humains et non humains sur lesquels reposent mouvements et ancrages. D'un point de vue méthodologique, Sheller et Urry (2006) proposent, en prônant l'émergence d'un « paradigme des nouvelles mobilités ", de combiner les méthodes des différents champs de recherche scientifiques et techniques s'intéressant aux mobilités et aux migrations. Dans la lignée de ces travaux, nous cherchons à prendre en considération la mobilité artistique dans sa pluridimensionnalité, à partir du croisement des deux corpus évoqués en introduction.

\section{Portrait des artistes en « mobiles avancés »}

7 Les artistes comptent parmi les «mobiles avancés » (Elliott, Urry, 2010), se déplaçant « entre expositions, foires artistiques et période de résidence, dans un marché de l'art globalisé, ils explorent aussi les conditions et les formes du voyage, les géographies et les politiques de la mobilité » (Witzgall, 2013, p. 7). Les travaux récents consacrés à ces mobilités permettent à la fois de mieux comprendre leurs enjeux politiques, économiques et culturels et d'en relativiser la portée généralisante et essentialisante (Boichot, 2012 ; Favell, 2008).

8 La mobilité des artistes et des œuvres constitue tout d'abord un enjeu politique qu'ont bien saisi les acteurs des politiques culturelles (Saez, Saez, 2012). Outils de promotion territoriale, vecteurs d'échange et de coopération, synonymes d'attractivité locale, les mobilités sont pleinement intégrées aux politiques culturelles européennes, à tous les échelons. Malgré tout, les travaux consacrés aux enjeux territoriaux des politiques culturelles ne les abordent que rarement de manière centrale (Amilhat-Szary et al., 2010).

9 Les enjeux économiques et politiques des mobilités artistiques apparaissent ensuite de manière plus centrale dans les théories de la ville et de la classe créatives (Florida, 2002; Landry, 2000). Ces théories, largement débattues et critiquées (Peck, 2005 ; Vivant, 2006), continuent à inspirer les politiques urbaines, notamment pour accroître la compétitivité et l'attractivité des territoires. Or cette attractivité se manifesterait justement par la mobilité de la classe créative - dont les artistes comptent au noyau dur selon R. Florida vers les lieux, et surtout les lieux urbains, offrant le cadre de vie le plus propice au développement de la créativité (Florida, 2005). Les études récentes contredisent toutefois l'hypothèse d'un lien causal entre l'attractivité des villes et la mobilité des membres de la classe créative (Martin-Brelot et al., 2010).

10 Les enjeux des mobilités artistiques sont aussi culturels et symboliques. Les mobilités artistiques, à l'instar de l'ensemble des formes de mobilités, supposent l'acquisition de compétences mobilitaires et l'apprentissage de codes et de normes culturels liés aux voyages et aux lieux fréquentés (Kaufmann, 2001). Mais une des spécificités des arts réside aussi dans une longue culture du voyage comme vecteur d'acquisition de 
compétences techniques et relationnelles directement liées à l'activité artistique. L'apprentissage - de nouvelles techniques, d'approches esthétiques différentes, de codes sociaux et relationnels - est ainsi l'un des motifs principaux de mobilité des artistes, avancé dès les prémices de l'institutionnalisation de la production artistique aux débuts de la Renaissance (Castelnuovo, Ginzburg, 1981 ; Heinich, 1996).

Enfin, les pratiques de mobilité sont porteuses de dynamiques d'hybridation: cultures locales et individuelles sont en effet susceptibles de se mêler en lien avec la mobilité des personnes, des biens et des informations (Bhabha, 1994 ; Hannerz, 1996). L'augmentation des mobilités et le développement des technologies de communication produisent de nouvelles esthétiques. Proches des esthétiques migratoires et des phénomènes de créolisation qui les caractérisent, ces "esthétiques mobiles" (Barriendos Rodriguèz, 2012) invitent à interroger "l'implication des politiques 5 de mobilité dans les subjectivités contemporaines» (op. cit., p. 342). J. Barriendos défend l'argument selon lequel, si la dimension symbolique de la mobilité est aujourd'hui prise en considération, il reste nécessaire de considérer cette « subjectivité mobile » d'un point de vue postcolonial, loin du mythe d'une culture globale, multiculturelle et transnationale. L'altérité est donc au cœur de l'analyse des mobilités dans le champ artistique, et est directement reliée à la question de l'émotion et de la corporéité.

Cette dimension corporelle et émotionnelle de la mobilité constitue une clé de lecture dans l'analyse des mobilités artistiques que nous proposons ici et nous permet d'interroger les effets de la confrontation à l'altérité du point de vue des individus et du point de vue des représentations collectives de soi et de l'autre dont les discours peuvent se révéler porteurs (Thrift, 2007).

\section{Des artistes mobiles aux spatialités en mouvement}

\section{Le déplacement : entre pratiques et espaces}

13 L'une des particularités de la mobilité des artistes est de s'inscrire dans une longue tradition (Bara, 2014 ; Guichard, 2010), tradition qui a pour effet, aujourd'hui, à la fois de valoriser cette pratique et de faire du voyage en soi l'intérêt du départ et de la mobilité. Cette idée est exprimée par Gilles Rhode, cofondateur de la compagnie d'art de la rue Transe Express, lorsqu'il commence le récit des voyages de la compagnie par :

«Le voyage est dans les gènes des saltimbanques; il est ferment et aboutissement

de leurs aventures ». (Rhode, 2013, p. 50)

14 Cette citation montre à quel point la pratique du voyage peut être intégrée dans l'imaginaire des artistes, en particulier dans le domaine du spectacle vivant. Il est un "ferment », une source de motivation, mais en étant un «aboutissement », il peut aussi devenir un mode de vie itinérant qui trouve sa raison d'être dans un déplacement récurrent au service de la créativité ou de la diffusion. Cet imaginaire du voyage allié à la pratique du déplacement produit ainsi des spatialités qui privilégient le temps et les espaces du mouvement.

Le musicien Renaud Brustlein revendique cette appartenance à un monde de la route. Il dit avoir besoin de se sentir en mouvement, que cela le plonge dans un «état d'esprit contemplatif », lui procure un équilibre et sert sa créativité. Lorsqu'il parle de son album off the map, il évoque le rapport à l'espace forgé au cours de ses multiples tournées en France mais aussi en Espagne, en Amérique du Nord, en Belgique ou en Suisse : 
" Je me suis carrément servi du fait d'aller à l'étranger pour prendre du recul et de la hauteur à tous les sens du terme, [...] ça m'a permis d'adopter une espèce de point de vue plus aérien comme si je me plaçais comme un satellite au-dessus, quoi, et que j'observais les trajectoires mais beaucoup plus grandes.» Entretien avec Renaud Brustlein (octobre 2013).

Il ressort du discours de l'artiste une spatialité imprégnée par une impression de fluidité et de trajectoires tous azimuts, les siennes ainsi que celles des êtres et objets mobiles qui l'entourent, et qui l'amènent à concevoir l'ensemble de ses déplacements avec « une espèce de point de vue plus aérien » (op. cit.).

À une autre échelle et dans le secteur des arts visuels, Nicolas Aiello investit la pratique et les espaces du déplacement dans les villes. Il collecte des images, des mots lors de marches dans la ville. Ces marches font elles-mêmes partie du processus de création puisqu'elles sont à la fois une manière d'appréhender un espace inconnu, ou du moins non pratiqué, et un moyen de collecter les éléments nécessaires à un projet dépassant le seul espace de la marche. Des paysages urbains photographiés de manière systématique lors de marches à New York, à Aulnay, Paris ou Marseille sont par exemple à l'origine de la série $\mathrm{Rtf}^{6}$. Les marches et le corps en mouvement participent pleinement de la production des œuvres et placent le déplacement au cœur du processus de création.

Au-delà de l'investissement de l'espace en mouvement sous une forme esthétique, la distance, le nombre et les rythmes de déplacements produisent leurs propres repères et référents spatiaux qui révèlent des tensions entre les lieux et les espaces en mouvement. C'est particulièrement le cas lors de tournées longues et récurrentes qui produisent un désancrage aux lieux chez les artistes (Bourdeau, 2009 ; Le Menestrel, 2012). Le récit de Renaud Brustlein le confirme, le mouvement permanent des tournées produit une spatialité qui se porte davantage sur des rythmes que sur des lieux. Autrement dit, la route implique une routine qui repose sur des repères standardisés autour de micro-lieux (scène, loges, hôtels) et de rencontres très éphémères (public, équipes techniques). De ce fait, les espaces auxquels les artistes se rattachent sont les espaces du mouvement, en particulier leur moyen de locomotion, à l'image du camion d'H-Burns, groupe de R. Brustlein :

« Après, dans ton mouvement, tu recrées aussi une espèce de maison. Par exemple, on a acheté un camion de tournée et dedans, on a fait en sorte que les conditions de voyage soient le plus agréable, on regarde des films, on a une petite chaîne hi-fi, on s'est fait une petite table de salon à l'intérieur ».

Les lieux de destination sont, a contrario, vidés de toute contenance :

« tu vas toujours d'un point $\mathrm{A}$ à un point $\mathrm{B}$ sans jamais vraiment te poser. »

Cette spatialité du mouvement qui fait référence à une forme de nomadisme voire d'errance doit cependant être nuancée à plusieurs égards.

21 Premièrement, les voyages d'artistes ne se résument pas aux tournées longues et récurrentes. En effet, l'étude des différents projets retenus par le FIACRe montre que près de deux tiers d'entre eux portent sur des voyages qui enchaînent au moins deux destinations mais seulement un tiers de ces voyages sont des tournées longues, cumulant de six à vingt-cinq destinations. Deuxièmement, si la pratique de la tournée n'est pas exclusivement réservée au domaine du spectacle vivant, les exemples que nous avons évoqués précédemment nous portent à penser qu'elle en est quand même l'apanage, en particulier dans le secteur des musiques actuelles. En effet $90 \%$ des projets de tournées sont portés par des artistes du spectacle vivant et la totalité des tournées longues est 
portée par des musiciens, danseurs ou comédiens. Notons toutefois que quelques projets de voyages itinérants (qui représentent $10 \%$ ) sont portés par des arts visuels : art numérique, art audiovisuel et bande dessinée. Enfin, les voyages des artistes ne se résument pas à la figure essentialisante de la tournée ou même du voyage itinérant. Bon nombre de voyages d'artistes sont ponctuels, de courte durée ou privilégiant seulement une ou deux destinations. Ainsi, près de la moitié des projets du FIACRe ont pour but une résidence ou un stage de création ou de partage d'une pratique artistique. Ces projets, même s'ils peuvent se dérouler sur jusqu'à quatre destinations différentes, impliquent, par le temps passé dans les lieux qui peut aller d'une semaine à deux mois, et par les activités réalisées sur place, des spatialités bien plus ancrées que mouvantes. On peut en conclure qu'au-delà de la pratique du déplacement et de l'investissement dans la spatialité qu'elle induit, c'est la capacité à être mobile qui est largement partagée et exprimée par les artistes, plus qu'une itinérance voire un nomadisme systématiquement mis en pratique.

\section{Les « ailleurs »: des ancrages dans la mobilité}

Quelle que soit l'importance qui lui est accordée, la mobilité d'artiste est tendue vers un lieu, une destination, un « ailleurs ». Les artistes interrogés mentionnent avant tout leurs pratiques de mobilité en lien avec le lieu qu'ils désirent atteindre, lieu toujours mis en avant pour les caractéristiques singulières qu'il offre dans le cadre de la pratique artistique. C'est cet «ailleurs » qui, pour ce qu'il représente ou par les ressources qu'il propose, s'oppose à l'« ici» de l'artiste et devient moteur de sa mobilité. Cette mise en avant du lieu de destination montre l'importance de penser les pratiques de mobilité en système, dans la tension entre ancrages et mobilités qui structure les espaces de vie des individus et, dans notre cas, les spatialités artistiques (Boichot, 2012; Hirschhorn, Berthelot, 1996).

Le corpus du FIACRe nous donne un aperçu des lieux que les artistes ont l'intention de fréquenter dans le cadre des projets présentés. On remarque ainsi que la quasi-totalité des projets s'établit dans des lieux dédiés à l'art et la culture. Il s'agit de lieux de diffusion (salles de spectacle, d'exposition, musées, festivals), de lieux de création (lieux dédiés aux résidences d'artistes), d'institutions (universités, écoles d'art) et puis des lieux de la représentation culturelle française à l'étranger (Instituts français et Alliances françaises). Quelques projets (qui représentent moins de 10\%) ont néanmoins pour destination des lieux qui ne sont pas, a priori, dédiés à l'art et la culture, mais plutôt la ville en elle-même, une friche industrielle, un quartier en particulier... Ces projets sont clairement motivés par la rencontre d'une altérité, à la fois sous forme de personnes, de cultures, de paysages, de matériaux.

Le choix de la destination et le type de lieu visé mettent en évidence le lien entre les caractéristiques du lieu et l'objectif artistique du voyage. Nous constatons ainsi, à partir de la diversité des projets retenus par le FIACRe et au travers des entretiens menés auprès de plasticiens montreuillois et berlinois que les voyages d'artistes se répartissent en deux grandes familles de motifs: la diffusion d'œuvres ou de spectacles, d'une part, et la création d'œuvres ou de spectacles, d'autre part. Pour les projets du FIACRe, la totalité des projets revendique l'un de ces deux motifs et quelques-uns (20\%) revendiquent les deux à la fois. Pourtant, d'autres motifs existent et viennent se greffer à des voyages qui ont pour but premier de la diffusion ou de la création. Ainsi, la quasi-totalité des projets 
est assortie de moments soit de collecte de matériaux, soit de rencontres professionnelles dédiées au développement de la carrière de l'artiste ou de la compagnie (rencontre avec des partenaires, montage de projet, négociation en vue de futures diffusions...) soit, enfin, de moments de transmission ou d'échanges artistiques (stages, ateliers, master classes, séminaires...). Au regard de ces motifs et de leur articulation au sein des voyages, il apparait que le déplacement vers un lieu est d'abord bien souvent fonction des ressources offertes par celui-ci. Il peut s'agir de ressources institutionnelles - des contacts professionnels ou la mise à disposition de fonds - intégrées à des circuits artistiques que l'on peut, là aussi, qualifier d'institutionnels et qui s'organisent autour de lieux, d'acteurs, d'appels d'offres. Les ressources offertes dépendent aussi des projets et des parcours; et un paysage source d'inspiration, la proximité de réseaux professionnels personnels ou la nécessité de collecter un matériau localisé, comme des photographies ou des paroles d'habitants comptent aussi parmi les ressources offertes par les lieux, dans leur singularité.

Pour distinguer les spatialités liées au rapport au lieu et à l'engagement de l'artiste que celui-ci suppose, on peut s'appuyer sur la distinction entre deux acceptions du concept de lieu, Topos et Chôra, tels que les a théorisées A. Berque (2003) et qui montre sa portée heuristique pour comprendre les spatialités des œuvres d'art (Volvey, HoussayHolzschuch, 2007). Le Topos, assimilé à un « récipient immobile» (Berque, 2003, p. 555) peut correspondre aux rapports à des lieux fréquentés de manière indifférente ou presque. Au contraire, des lieux investis et intégrés aux processus artistiques, qu'il s'agisse de processus de création ou de diffusion, mobilisent davantage un référentiel au lieu comme Chôra, comme "matrice et empreinte [qui témoigne d'un] lien ontologique indissoluble entre les lieux et les choses » (ibid.). La distinction et l'articulation de ces deux types de lieux dans les parcours des artistes apparaissent clairement dans le récit du metteur en scène Gilles Pastor. L'artiste sépare bien, d'une part, ses voyages liés à la diffusion, qui sont courts, ponctuels et qui le dirigent vers des festivals ou des théâtres à New York, Bruxelles ou Genève ; et d'autre part, ce qu'il désigne comme étant des voyages guidés par des "affinités" et qu'il fréquente de manière récurrente et durant des périodes conséquentes allant de la semaine à plusieurs mois. Sa spatialité, dans sa manière de vivre et d'investir les lieux, se trouve ainsi très duale : les lieux-Topos sont très peu évoqués et sont qualifiés de "climatisés "; en revanche, ses lieux-Chôra que sont la ville de Salvador de Bahia au Brésil et la maison du cinéaste Derek Jarman en Angleterre ont été largement parcourus, exploités et vécus de manière sensible et émotionnelle. L'expérience de Gilles Pastor à Salvador de Bahia témoigne surtout d'un ancrage momentané dans la carrière d'un artiste : cinq voyages - d'une durée moyenne d'un mois - en sept ans, une relation qui a commencé par une résidence Hors les murs de l'Institut français de trois mois et qui s'est soldée par la création et la diffusion de deux spectacles. L'attachement à la ville s'est fait à plusieurs titres : son histoire et sa culture (le rite du Candomblé, le métissage afro-brésilien), ses habitants (vidéo Jaïr autour d'un garçon de café), ses conditions climatiques et environnementales (chaleur, humidité, coucher de soleil), des artistes et opérateurs culturels locaux (le danseur Edu O. notamment). Tout comme Salvador de Bahia a constitué pour Gilles Pastor un point de repère pendant un temps de carrière, le photographe berlinois Herbert se rend au moins une fois par an en Californie, terre d'élection qui nourrit son iconographie depuis plusieurs années. Le lieu d'inspiration peut être pris comme exemple emblématique d'un rapport au lieu-Chôra très présent dans les discours des artistes rencontrés. Se déplacer pour trouver un « ailleurs » propice à la création est une des motivations majeures et spécifiques de la mobilité 
artistique. Mais la distinction entre Chôra et Topos n'est pas toujours évidente et l'on peut aisément imaginer des glissements de l'un à l'autre. Si l'on reprend l'exemple d'Herbert, l'attachement fort à la Californie qu'il a développé s'explique aussi par son parcours et la formation qui l'a au départ conduit là-bas. Cette relation artistique au lieu est donc relative à un parcours de vie et s'inscrit dans une relation plus globale à un ensemble de lieux qui forment un réseau.

\section{Les parcours : des révélateurs de spatialités poly-topiques}

Les liens entre les lieux conduisent en effet à une proximité de type topologique plus que topographique et permettent de saisir un espace de référence de la pratique artistique poly-topique (Stock, 2006) et réticulaire, plus que linéaire et continu. On observe ce type de rapport à l'espace de manière évidente dans le cas de certains artistes valorisant l'expérience d'une pluralité de lieux. C'est le cas de Juan, photographe à Montreuil, dont le parcours artistique international s'inscrit dans la lignée de son expérience migratoire et le conduit à mobiliser un référentiel cosmopolite pour décrire les nombreux lieux de vie et de voyage qui caractérisent son parcours :

"J'ai vécu en Uruguay jusqu'à 12,13 ans. J'ai vécu un an au Guatemala avec mes parents, puis en France, à Paris, dans la banlieue ouest. J'ai vécu à Londres aussi [...] J'ai beaucoup voyagé pour la photo, j'ai énormément voyagé [...] sur tous les continents, dans plein d'endroits, pour des reportages, pour des sujets photo. [...] J'ai des copains partout, j'ai plein d'endroits où je peux rester. » Entretien avec Juan (janvier 2010).

Si l'œuvre peut être le résultat d'une vie de voyages et d'une spatialité réticulaire de l'artiste, elle peut, à l'inverse, susciter pour sa création la constitution d'un réseau, la collaboration d'acteurs dans différents lieux et le voyage, voire le déménagement, des artistes d'un lieu à un autre. C'est le cas pour la compagnie de danse Lanabel et l'œuvre chorégraphique "Atropos ». La création de cette pièce est un véritable cheminement entre Grenoble, Bergen (Norvège), Darmstadt (Allemagne) et Ouagadougou (Burkina Faso) fait de coproductions, de temps d'immersion et de résidences (figure 1). 
Figure 1. Parcours de création de la pièce « Atropos » de la compagnie de danse Lanabel.

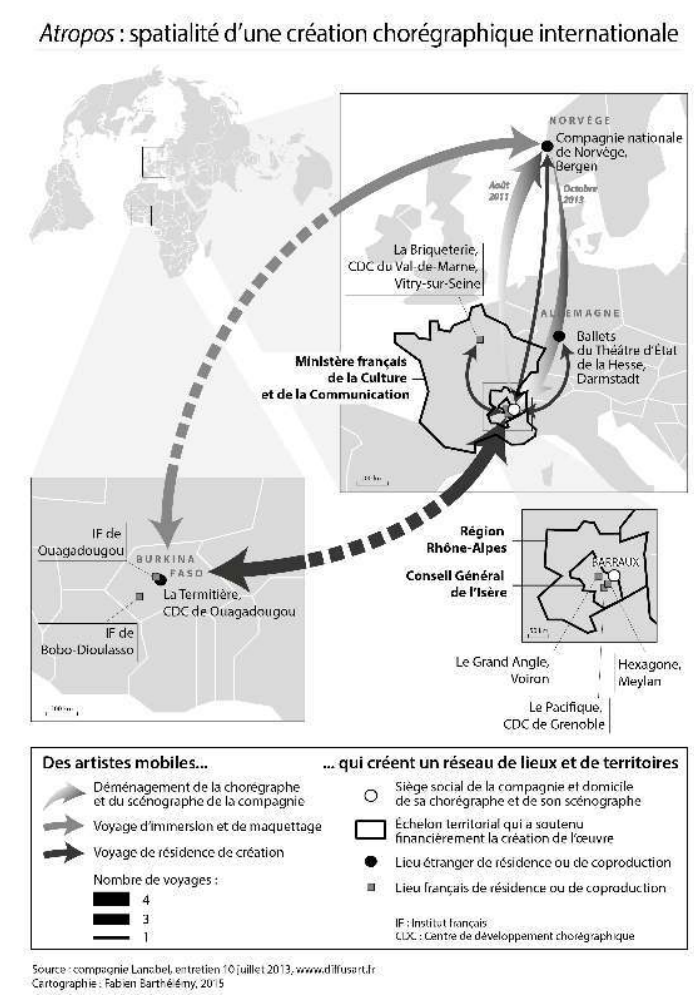

La mobilité, productrice de spatialités artistiques qui se comprennent dans le temps long du parcours des individus et dans la tension entre ancrages et circulation peut aussi devenir productrice de représentations spatiales intégrées aux œuvres, ce qui constitue un dernier élément des spatialités artistiques construites dans la mobilité que nous avons identifiées dans le croisement des deux corpus d'analyse.

\section{Intégrer l'altérité : les spatialités mobiles mises en œuvre}

Ce dernier point aborde la question des effets de la mobilité dans la production artistique en se penchant plus particulièrement sur la question de l'expérience migratoire et des productions artistiques auxquelles elle donne lieu, élément révélateur des processus d'hybridation symbolique et culturelle dont elle peut être porteuse.

Près de la moitié des projets soutenus par le FIACRe est à la base de collaborations artistiques entre partenaires de différentes nationalités et l'on peut distinguer plusieurs degrés de collaboration avec des artistes créateurs ou interprètes étrangers afin d'aboutir à une œuvre dont la production peut être qualifiée d'internationale. En particulier, quatre projets associent plusieurs lieux, plusieurs pays et plusieurs compagnies ou collectifs pour créer des plateformes de coopération artistique plurinationales. Dans ces projets, la mobilité est au service de la rencontre avec l'altérité: d'autres artistes, d'autres structures, d'autres savoir-faire, d'autres sujets de création, et l'argumentaire de ces projets repose sur l'assurance que la rencontre de ces différences sera fertile sur le plan artistique. Ces exemples de démarches artistiques internationales participent d'une 
volonté d'hybridation, de métissage et de la recherche d'une dimension cosmopolite qui est valorisée par les artistes (Le Menestrel, 2012).

31 L'intégration de l'autre dans le parcours artistique, qu'il soit de l'ordre du voyage ou de l'expérience migratoire, produit donc des effets dans les contenus et les techniques des œuvres. Ceux-ci dépendent évidemment de l'histoire et de la démarche de chacun mais plusieurs artistes témoignent, comme Natsuyo Koizumi ou la compagnie de danse Lanabel, de l'utilisation directe de leurs expériences migratoires dans leur création, notamment à travers une intégration consciente d'éléments culturels, esthétiques et mémoriels liés à ces expériences. Avec "Spreewald», Natsuyo Koizumi illustre l'hybridation de plusieurs cultures dont son univers visuel est le fruit (figure 2). Elle croise à la fois des techniques et une esthétique qui rappelle celle des estampes japonaises et la représentation du paysage naturel lacustre et forestier aujourd'hui patrimonialisé de la région berlinoise : celui de la Spreewald. L'œuvre est ainsi, pour l'artiste un moyen de :

«mettre en évidence la cohabitation culturelle et par là de découvrir une culture en elle-même singulière. Cette image représente aussi aujourd'hui une indication de la manière dont je pouvais [au moment de sa création] percevoir visuellement [le monde].»

Figure 2. « Spreewald. Eau forte».

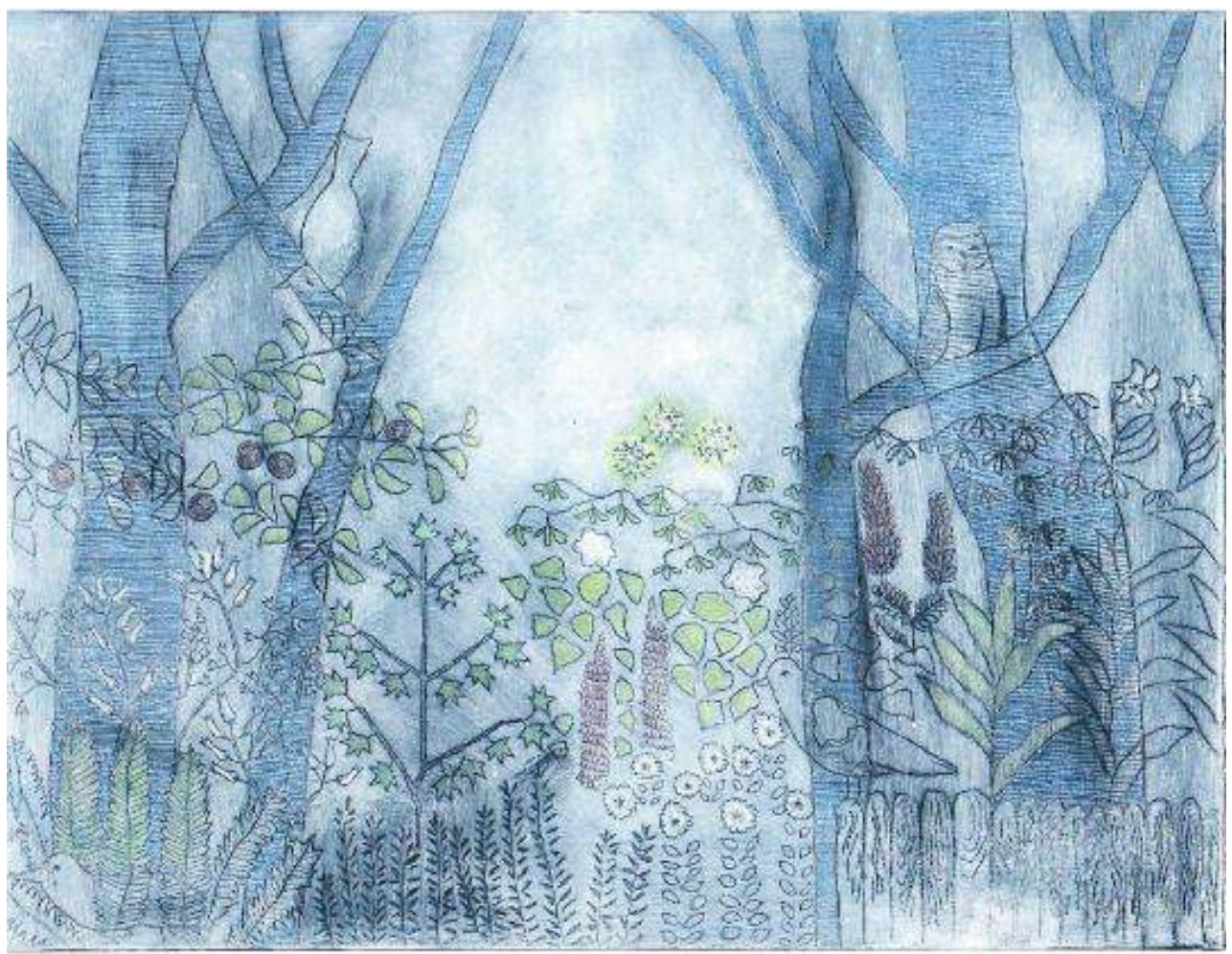

Source : Natsuyo Koizumi, 2012

Dans une temporalité qui est celle de la carrière, l'œuvre chorégraphique « Atropos » de la compagnie Lanabel se pose comme l'aboutissement de près de dix ans de voyages et de rencontres. Pour l'occasion, le propos est clairement tourné vers l'altérité et les échanges; la création a lieu en partie au Burkina Faso pour cette raison:

F.D. : « Après, là on rentre dans un processus d'une autre création qui est en lien avec le Burkina Faso où là, par contre, on va chercher l'atmosphère, enfin la rencontre là-bas mais en lien avec la création. » 
A.B. : «Ouais, donc là ça influencera la création complètement et là on va le chercher, volontairement, on se met dans cette situation-là, exprès. Donc c'est encore une autre démarche." Entretien avec Annabelle Bonnéry et François Deneulin de la compagnie Lanabel (10 juillet 2013). déplacement de statuettes africaines que la chorégraphie qui met en avant le dialogue des corps évoquent l'expérience, vécue en voyage, de la relation à l'autre et à l'ailleurs (figure 3).

Figure 3. Miroir et dialogue dans la chorégraphie d'« Atropos ».

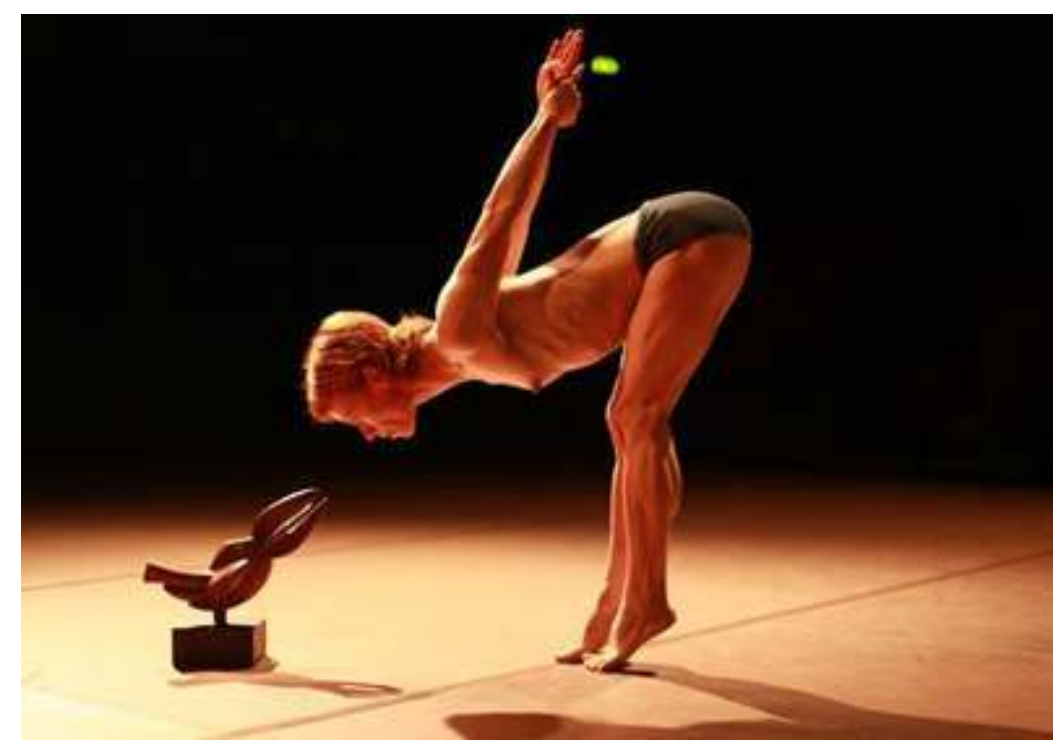

Photographie d'Antoine Conjard, source : Lanabel, 2015.

Plus qu'un effet de lieu à proprement parler, c'est la confrontation à l'altérité et l'hybridation culturelle dont les mobilités sont fréquemment porteuses que mettent en avant les discours des artistes. Il s'agit en effet d'un ressort important de la création que l'on retrouve de manière plus ou moins explicite dans les œuvres produites et qui montre comment des pratiques de mobilités à plus ou moins long terme peuvent marquer les pratiques comme les représentations spatiales des artistes, mais aussi du public qui reçoit les œuvres.

\section{Conclusion}

Faire dialoguer arts du spectacle et arts plastiques nous a permis de mettre en avant des spatialités artistiques accordant une place importante à la mobilité. L'un des premiers apports de cet article nous semble justement résider dans le dialogue entre disciplines artistiques que nous avons proposé à travers la mise en perspective de nos deux corpus. Ce croisement nous a permis de confirmer ce qui était à l'origine de notre volonté d'écriture à deux mains, à savoir que la mobilité joue un rôle prépondérant dans l'organisation des parcours professionnels des artistes au-delà des spécificités propres à chaque discipline artistique, qu'il s'agisse de l'art contemporain, de la danse, du cirque ou de la musique pour reprendre quelques-uns des exemples cités dans le texte. Nous avons mis l'accent sur ce qui nous semble être deux éléments caractéristiques des mobilités 
artistiques : la diversité des pratiques d'abord, où fréquence et durée des voyages peuvent être très variables d'une discipline et d'un artiste à l'autre, mais aussi et surtout la valorisation du voyage en lui-même comme un élément enrichissant pour la pratique professionnelle. De manière générale, nous avons montré que la mobilité constitue un puissant facteur d'organisation des espaces, permettant d'articuler entre eux les différents lieux et acteurs des arts.

Le second apport de cet article réside à notre sens dans l'accent mis sur le rôle d'articulation que joue la mobilité entre des ancrages artistiques pluriels. Loin d'un nomadisme artistique généralisé, nos observations montrent au contraire l'importance des lieux et de l'ancrage au cœur de ceux-ci, les spatialités des artistes rencontrés dessinant au final des réseaux de lieux dynamiques. La " poly-topie » apparaît, à la lumière des discours recueillis, comme une ressource très fréquemment mobilisée pour mener une carrière d'artiste professionnel. La tension entre ancrage et mouvement mise en avant dans les travaux sur la mobilité nous semble finalement accentuée dans le cas des artistes par l'attention portée au fait d'être en mouvement et par le rapport sensible aux lieux.

Le troisième et dernier apport de cette réflexion sur la mobilité artistique réside dans la mise en avant de l'utilisation de la mobilité et de ses effets de décentrement et de confrontation à l'altérité dans les productions artistiques. Le mouvement et les ancrages multiples nourrissent fréquemment les œuvres et les mobilités artistiques se traduisent par un effet direct en terme de production de représentations des espaces perceptibles dans les œuvres, qu'il s'agisse de peinture de paysages mêlant les caractéristiques de plusieurs lieux fréquentés par le peintre ou bien de paroles de chansons reflétant la multiplicité des ancrages. Nous apportons au final une réflexion empirique sur le rôle des mobilités dans l'organisation des espaces de la production artistique qui relativise largement l'archétype d'un artiste nomade, mais qui met en avant le rôle des pratiques de mobilité, aussi diverses soient-elles dans un rapport singulier et sensible à des espaces multiples.

\section{BIBLIOGRAPHIE}

AMILHAT-SZARY A.-L., LOUARGANT S., KOOP K., SAEZ G. (2010), Artists Moving and Learning. A comparative study on artistic mobility, European Commission.

BARA O. (2014), « Vedettes de la scène en tournée: première mondialisation culturelle au XIXe siècle? ", Romantisme, 163, 1, pp. 41-52.

BARRIENDOS RODRIGUÈZ J. (2012), « Global Art and the Politics of Mobility: (Trans)Cultural Shifts in the International Contemporary Art-System ", in Bal M. \& Hernadez-NavarroM. (dir.), Art and Visibility in Migratory Culture: Conflict, Resistance, and Agency, Amsterdam, Rodopi, pp.

313-334.

BERNIER X. (2013), « Traverser l'espace », EspacesTemps.net, Travaux. 
BERQUE A. (2003), « Lieu », in Lévy J. \& Lussault M. (éd.), Dictionnaire de la géographie et de l'espace des sociétés, Paris, Belin, pp. 555-556.

BHABHA H. K. (1994), The Location of Culture, London, Routledge.

BOICHOT C. (2012), Centralités et territorialités artistiques dans la structuration des espaces urbains: le cas de Paris et de Berlin, Thèse de doctorat, Université Panthéon-Sorbonne, Paris. BOISSIÈRE A., FABBRI V., VOLVEY A. (2010), Activité artistique et spatialité, Paris, L'Harmattan. BOURDEAU P. (2009), « Les Rolling Stones en tournées », La Géographie, 6, pp.84-87.

CASTELNUOVO E., GINZBURG C. (1981), « Domination symbolique et géographie artistique », Actes de la recherche en sciences sociales, 40, 1, pp. 51-72.

CORDIER M. (2007), « Le cirque contemporain entre rationalisation et quête d'autonomie », Sociétés contemporaines, 66, 2, pp.37-59.

CRESSWELL T. (2006), On the move : mobility in the modern Western world, New York, Routledge. DE MORANT A. (2007), Nomadismes artistiques : des esthétiques de la fluidité, Thèse de doctorat, Université Paris Ouest Nanterre, Paris.

DENIAU M. (2011), Les échanges entre la France et l'Europe, Paris, Office National de Diffusion Artistique.

ELLIOTT A., URRY J. (2010), Mobile Lives, London, Taylor and Francis.

FAVELL A. (2008), Eurostars and Eurocities: Free Movement and Mobility in an Integrating Europe, Oxford, Wiley-Blackwell.

FLORIDA R. (2002), The Rise of the Creative Class: And How It's Transforming Work, Leisure, Community and Everyday Life, New York, Basic Books.

FLORIDA R. (2005), The flight of the creative class: The new global competition for talent, NewYork, Harperbusiness.

GUICHARD C. (2010), « Les circulations artistiques en Europe (années 1680-années 1780) », in Beaurepaire P.-Y., Pourchasse P., Les circulations internationales en Europe, années 1680-années 1780, Rennes, Presses Universitaires de Rennes, pp. 385-398.

HANNERZ U. (1996), Transnational Connections: Culture, People, Places, London, Routledge.

HEINICH N. (1996), Être artiste. Les transformations du statut des peintres et des sculpteurs, Paris, Klincksieck.

HEINICH N. (2005), L'élite artiste: excellence et singularité en régime démocratique, Paris, Gallimard.

HIRSCHHORN M., BERTHELOT J.-M. (dir.) (1996), Mobilités et ancrages. Vers un nouveau mode de spatialisation?, Paris, L'Harmattan.

KAUFMANN V. (2001), « La motilité : une notion clé pour revisiter l'urbain », in Bassand M., Kaufmann V., Joye D. (dir.), Enjeux de la sociologie urbaine, Lausanne, Presses polytechniques et universitaires romandes, pp. 87-102.

KAUFMANN V. (2002), Re-thinking mobility, Adlershot, Ashgate.

KAUFMANN V. (2008), Les paradoxes de la mobilité: bouger, s'enraciner, Lausanne, Presses polytechniques et universitaires romandes. 
LANDRY C. (2000), The creative city: a toolkit for urban innovators, Oxford, Earthscan

Publications Ltd.

LE MENESTREL S. (2012), Des vies en musique: parcours d'artistes, mobilités, transformations, Paris, Hermann.

MARTIN-BRELOT H., GROSSETTI M., ECKERT D., GRITSAI O., KOVÀC Z. (2010), « The Spatial Mobility of the 'Creative Class': A European Perspective », International Journal of Urban and Regional Research, 34, 4, pp.854-870.

MENGER P.-M. (2002), Portrait de l'artiste en travailleur: métamorphoses du capitalisme, Paris, Seuil.

PECK J. (2005), « Struggling with the Creative Class », International Journal of Urban and Regional Research, 29, 4, pp. 740-770.

RETAILLÉ D. (2009), « Malaise dans la géographie, l'espace est mobile », in VANIER M. (dir.), Territoires, territorialité, territorialisation: controverses et perspectives, Rennes, Presses Universitaires de Rennes.

RHODE G. (2013), « Rêves d'adolescent », Stradda, 29, pp.50-51.

SAEZ G., SAEZ J.-P. (dir.) (2012), Les nouveaux enjeux des politiques culturelles : dynamiques européennes, Paris, La Découverte.

SHELLER M., URRY J. (2006), « The new mobilities paradigm », Environment and Planning A, 38, 2, pp.207-226.

STOCK M. (2006), « L'hypothèse de l'habiter poly-topique : pratiquer les lieux géographiques dans les sociétés à individus mobiles ", EspacesTemps.net.

THRIFT N. (2007), Non-Representational Theory: Space, Politics, Affect, London, Routledge. URRY J. (2000), Socioloy Beyond Societies. Mobilities for the Twenty-First Century, London, Routledge.

VIVANT E. (2006), « La classe créative existe-t-elle? », Les annales de la recherche urbaine, 101, pp.155-161.

VOLVEY A., HOUSSAY-HOLZSCHUCH M. (2007), « La rue comme palette. », Travaux de l'Institut de Géographie de Reims, 129-130, pp.145-174.

WITZGALL S. (2013), « Mobility and the image-based research of art ", in WITZGALL S., VOGL G., KESSELRING S., New Mobilities Regimes in Art and Social Sciences, London, Ashgate, pp. 7-16.

ZELINSKI W. (1971), « The Hypothesis of the Mobility Transition », Geographical review, 61, 2, pp. 219-249.

\section{NOTES}

1. Nous considérons que la dimension internationale est pertinente dans la mesure où elle impose un cadre de franchissement, en l'occurrence celui de frontières nationales.

2. Fonds d'innovation artistique et culturel de Rhône-Alpes.

3. Ville et arrondissement respectivement situés à l'est de la petite couronne parisienne et au sud-est de Berlin. 
4. Peinture, sculpture, installation, photographie, vidéo, livre et bande dessinée, musiques actuelles, musiques classiques et contemporaines, théâtre, danse, arts de la rue, cirque, sont ainsi représentés.

5. Le terme renvoie à la notion anglaise politics qui comprend le politique dans un sens étendu.

6. http://nicolasaiello.com/

\section{RÉSUMÉS}

Quelle commune mesure y a-t-il entre les Rollings Stones en tournées planétaires et un plasticien qui vient s'installer dans un quartier gentrifié d'une capitale culturelle comme Berlin? Cette question nous a amenés à penser la mobilité artistique de manière large en prenant en compte la diversité des pratiques de mobilité et en dépassant les frontières disciplinaires de l'art. C'est pourquoi nous basons notre étude sur le croisement de deux corpus qui mêlent les arts visuels et le spectacle vivant et qui proposent l'un, une dimension descriptive issue d'une base de données et l'autre, une dimension compréhensive issue d'entretiens. Ce parti pris méthodologique nous permet d'aborder la question des mobilités artistiques sous l'angle des spatialités du mouvement qui mettent en avant une articulation entre ancrage et mobilité, entre voyages et immersions dans des lieux.

What do the Rolling Stones' worldwide tours and a visual artist who is coming to settle in a gentrified place of a cultural capital such as Berlin have in common? This question led us to think artistic mobility with a broad sense, taking into account the diversity of practices and going beyond art's disciplinary boundaries. That is why our study combines two complementary corpuses on visual arts and performing arts: the first one offering a general overview of artists' mobilities through an extensive database; the second one constituted from interviews, allowing for an in-depth understanding of artists' motivations. With this methodological choice, we can tackle the topic of artistic mobility focusing on spatialities on the move that stress out the connection between routes and roots, between travels and immersion into places.

\section{INDEX}

Keywords : artists, visual arts and performing arts, international mobility, spatialities

Mots-clés : artistes, arts visuels et spectacle vivant, mobilités internationales, spatialités

\section{AUTEURS}

\section{FABIEN BARTHÉLÉMY}

PACTE UMR 5194, Univ. Grenoble Alpes, Grenoble, France, fabien.bart@orange.fr

\section{CAMILLE BOICHOT}

Géographie-Cités UMR 8504, Paris, France, camille.boichot@gmail.com 